\title{
Cerebrotendınous xanthomatosıs: a case report
}

\begin{abstract}
A 19 year old female presented with painfull swelling in bilateral Achilles tendon, juvenil bilateral cataracts and hypercholesteremia. On physical examination, there were firm and fixed swelling with an average diameter between 2 and $3 \mathrm{cms}$, on posterior aspect of the both ankles. Magnetic resonance imaging revealed bilateral enlarged Achilles tendons in both ankles and abnormalities in supraventricular white matter of the left frontal and parietal lobes of brain. The patient was diagnosed as cerebrotendinous xanthomatosis based on the clinical and radiological features.
\end{abstract}

Cerebrotendinous xanthomatosis is a rare autosomal-recessive disorder due to mutations in the CYP27A1 gene which encodes the mitochondrial enzyme sterol 27-hydroxylase. It is clinically characterized by cataracts, diarrhoea, xanthomas, progressive neurological symptoms, premature arteriosclerosis and coronary heart disease. The progress of cerebrotendinous xanthomatosis can prevent with early diagnosis and treatment.

Keywords: cerebrotendinous xanthomatosis, xanthoma, congenital cataract
Volume 2 Issue 3 - 2017

\author{
Gulnur Tasci Bozbas, Omer Faruk Sendur, \\ Mürüvvet Nalbantoğlu \\ Department of Physical Medicine and Rehabilitation, Adnan \\ Menderes University Medical Faculty, Turkey
}

\begin{abstract}
Correspondence: Gulnur Tasci Bozbas, Physical Medicine and Rehabilitation Department,Adnan Menderes,University Medical School,Aydin, Turkey, Tel 0090-505-83।3224,
\end{abstract}

Email gulnurtb@yahoo.com

Received: September 25, 2017 | Published: November 24, 2017

\section{Introduction}

Cerebrotendinous xanthomatosis is a rare autosomal recessive disorder. ${ }^{1}$ In this article, we reported a case of CTX presenting with bilateral Achilles tendon xanthomas along with juvenil cataracts and hypercholesteremia. We aimed to draw attention to the CTX in this report. Physicians should consider the CTX in patients presenting with combination of xanthomatosis, juvenile cataracts and neurological symptoms for early diagnosis and treatment of these patients can reduce mortality and morbidity. ${ }^{1}$

\section{Case}

19-year-old woman presented with pain and swelling at back of the ankle joint over one month (Figure 1). She reported diffuculty in walking. She had no history of injury, trauma or infection. She had sickle cell anemia trait and bilateral cataract surgery at eight years old. On physical examination, there were firm and fixed swelling with an average diameter between 2 and $3 \mathrm{cms}$, bilaterally on the posterior aspect of the ankle. Bilateral Achilles tendons were tender. Other general and systemic examinations were normal.

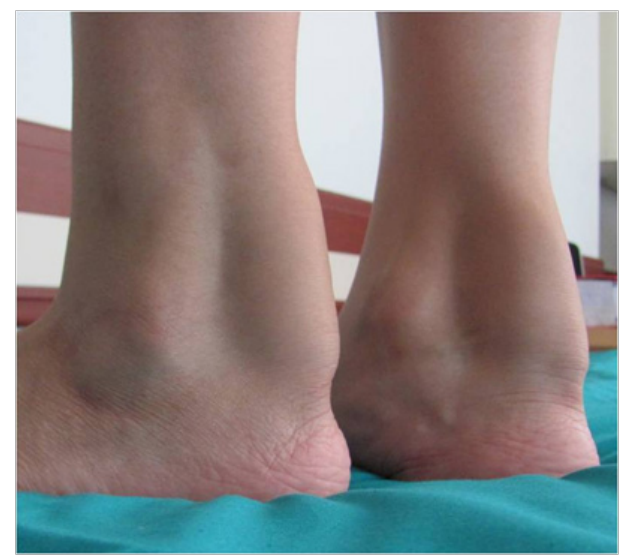

Figure I Bilaterally swelling on the posterior aspect of the ankle.
Laboratory tests revealed a normal complete blood count, inflamatuary markers and serological tests. Lipid profile revealed serum total cholesterol $269 \mathrm{mg} / \mathrm{dl}$, serum triglyceride $97 \mathrm{mg} / \mathrm{dl}$, serum LDL cholesterol 144mg/dl and serum HDL cholesterol $106 \mathrm{mg} /$ dl. Other blood parameters were within normal limits. Plain lateral radiographs of the ankles revealed bilateral soft tissue opacities in the region of the Achilles tendon without bone pathologies. In magnetic resonance imaging (MRI) of both ankles revealed bilateral enlarged Achilles tendons showing hypointensities on T1-weighted sequences and hyperintensities on T2-weighted and short T1 inversion-recovery (STIR) sequences. These findings were consistent with xanthoma (Figure 2). Cranial MRI revealed abnormal signal intensity characterized by hypointense T1-weighted and hyperintense T2-weighted echo images on supraventricular white matter of the left frontal and parietal lobe. MRI of sacroiliac joints were normal. ECG findings were within normal limits. It was thought that the diagnosis of the patient is CTX because on patient history, clinical and radiological findings. She was treated with ursodeodeoxycholic acid $(750 \mathrm{mg} /$ day). After 3 months of the treatment, cholesterol levels were back to normal, decreased swelling and completely healed the pain in Achilles tendon.

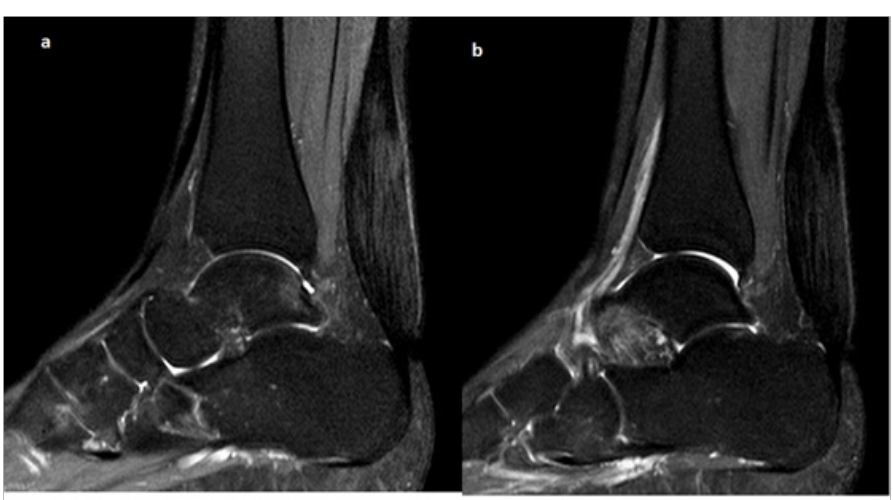

Figure 2 MRI of both ankles; revealed bilateral enlarged Achilles tendons showing hyperintensities on short TI inversion-recovery (STIR) sequences. 


\section{Discussion}

Cerebrotendinous xanthomatosis is a very rare genetic disorder. Only about 300 cases reported worldwide up to the present. ${ }^{2}$ In patients with CTX, cholesterol cannot be converted into bile acids (cholic acid and chenodeoxycholic acid) due to mutations in the gene CY27A1, which encodes the mitochondrial enzyme sterol 27-hydroxylase, but is instead converted into cholestanol and bile acid. ${ }^{1}$ Subsequently cholestanol accumulates in various tissues predominantly within the central nervous system, tendons, liver, lung and kidneys. Defective bile acid leads to increased incidence of cholelithiasis. ${ }^{2}$ Cerebrotendinous xanthomatosis may occur differrent clinical presentation due to multiorgan involvement. These symptoms are a broad range of as neurological and non-neurological. The disease is characterized by diarrhea and bilateral cataracts in childhood period. ${ }^{1}$ In more advanced stages, the most common symptoms are tendon xanthomas and signs of progressive neurologic dysfunction such as ataxia, paraparesis, cognitive impairment, psychiatric disorders, Parkinson's disease, polyneuropathy, epilepsy. Less frequently, coronary heart disease and atherosclerosis has been defined. ${ }^{3}$

In our case, the patient is a 19 year old female, who presented with bilateral ankle swelling, juvenil cataracts and hypercholesteremia. Neurological symptoms has not been developed yet. Diagnosis of CTX is based on clinical and radiological findings. Clinical findings is very important in diagnosis of CTX. Radiological and biochemical findings are necessary in young patients with early disease signs, such as cataract or diarrhea ${ }^{1,2}$ Magnetic resonans imaging of the brain shows focal/ diffuse white matter abnormalities and different degrees of cerebral and cerebellar atrophy in patients with CTX. ${ }^{2}$ In our case, white matter abnormalities in MRI were determined. This finding was determined in $71-100 \%$ of patients with CTX. The hyperintense signals in dentate nuclei, globus pallidus and pyramidal tract at MRI T2/ FLAIR are other characteristic findings of CTX. ${ }^{2}$ In biochemical examination of patients with CTX, high serum cholesterol and cholestanol levels can be determined. Pilo de la Fuente was determined normal serum cholestenol level in eight of 14 CTX patients who have xanthomas. ${ }^{3}$ When the literaturs are evaluated, it was determined that serum cholesterol levels in patients of CTX can be normal, low or high. ${ }^{2-4}$ As a result high cholesterol and cholestenol levels is important in the diagnosis of CTX, but normal values does not rule out this disease. ${ }^{2-5}$ Nevertheless it is lack of in our case that the serum cholestenol was not evaluated. The clinical, biochemical and genetic findings is important determination of CTX in young patients with early disease signs, such as juvenile bilateral catarac. However in advanced stage, the diagnosis of disease can be made by clinical and radiological evaluation, as in our case. ${ }^{1,2}$
Xanthoms seen CTX can interfere with enthesitis related ankylosing spondylitis, rheumatoid nodules, musculoskeletal tumors. ${ }^{2-5}$ In our case, the differential diagnosis in these disorders was made by serological and radiological evaluations. Clinically, CTX resembles to familial hypercholesterolemia or hyperlipoproteinemia, but biochemical analysis reveals that these disorders contain high amounts of cholesterol. ${ }^{5}$ In the presense of neurological findings, CTX resembles Myotonic dystrophy type I, spinocerebellar degeneration, spastic paraplegia and Parkinson's disease but can differentiated with the presence of tendon xanthomas. ${ }^{2}$

The management of CTX is replacement therapy which consist of administration of bile acids such as, chenodeoxy cholic acid, ursodeoxycholic acid, cholic acid, or taurocholic acid..$^{1,3}$ Bile acid supplementation can restore bile acid synthesis by reducing plasma cholestanol levels and eliminating bile alcohols. ${ }^{1}$ Management with bile acid either alone or association with $\mathrm{HMG}-\mathrm{CoA}$ reductase inhibitors should be started the early stages of the disease especially when neurological findings is not established. In this condition, the neurologic dysfunction associated with this disease may be prevented or even improved. ${ }^{1}$ Clinical improvement takes time and the patient must be advised long-term follow-up and periodic evaluation.

\section{Acknowledgements}

None.

\section{Conflict of interest}

The author declares no conflicts of interest.

\section{References}

1. Nie S, Chen G, Cao X, et al. Cerebrotendinous xanthomatosis: a comprehensive review of pathogenesis, clinical manifestations, diagnosis, and management. Orphanet J Rare Dis. 2014;26(9):179.

2. Pudhiavan A, Agrawal A, Chaudhari S, et al. Cerebrotendinous xanthomatosis-the spectrum of imaging findings. J Radiol Case Rep. 2013;7(4):1-9.

3. Pilo FB, Sobrido MJ, Girós M, et al. Usefulness of cholestanol levels in the diagnosis and follow-up of patients with cerebrotendinous xanthomatosis. 2011;26(7):397-404.

4. Huang L, Miao XD, Yang DS, et al. Bilateral achilles tendon enlargement. Orthopedics. 2011;34(12):960-964.

5. Sanjeev J, Mohd K, Kamlesh. Cerebrotendinous xanthomatosis, early diagnosis mandatory: Report of a case from North India. Neurology Asia. 2018;13:125-128. 